\title{
Attenuation and amino acid changes in infectious bronchitis virus Iranian 793/B serotype during serial passaging in embryonated chicken eggs
}

\author{
A. SALARPOUR ${ }^{1}$, R. TOROGHI ${ }^{1 *}$, G. R. NIKBAKHT ${ }^{2}$, A. REZAI ${ }^{1}$, R. MOMAYEZ $^{3}$
}

\begin{abstract}
${ }^{1}$ Department of Vererinary Research and Biotechnology, Razi Vaccine and Serum Research Institute, Mashhad, Iran;
${ }^{2}$ Department of Microbiology and Immunology, Veterinary faculty, Tehran University, Tehran, Iran;

${ }^{3}$ Department of Poultry Disease Research and Diagnosis, Razi Vaccine and Serum Research Institute, Karaj, Iran
\end{abstract}

Received September 25, 2018; revised March 27, 2019; accepted September 19, 2019

\begin{abstract}
Summary. - The use of live attenuated vaccine (LAV) is the main method for controlling infectious bronchitis (IB). It is advisable to develop a LAV using a dominant serotype in the region in the case of vaccine failure. Since 793/B serotype is one of the most predominant circulating IB viruses in Iran, attenuation of three Iranian 793/B isolates (IR/773/2001, IR/794/2002 and IR/520/2002) was done by serial passaging in specific pathogen free (SPF) embryonated chicken eggs up to 90 passages to assess the degree of their attenuation to achieve a native LAV in the future. Virulence and pathogenicity of passage levels 15 and 90 of isolates 773 and 794 were compared using histopathology, ciliostasis and potency tests. The results showed a decrease in the virulence and pathogenicity of the isolates at passage 90 compared to passage 15, although this decrease in pathogenicity was very mild and viruses after passage 90 were not adequately attenuated. Each isolate underwent some amino acid changes at passage 90 . In case of isolate 773 it was 5 aa changes, while in isolate 794 it was 19 aa changes. Some amino acid changes resulted in change into amino acid with different hydrophobicity characteristics. No amino acid change was found at passage level 15 compared to wild type viruses. Interestingly, we did not find previously reported change in amino acid 95 in passage levels 15 and 90.
\end{abstract}

Keywords: infectious bronchitis; live attenuated vaccine; 793/B serotype; pathogenicity; attenuation; nucleotide sequencing

\section{Introduction}

Avian infectious bronchitis virus (IBV) causes a highly infectious upper respiratory tract disease, nephritis and decreased egg production and egg quality (Jackwood et al., 2010; Tarpey et al., 2006). The virus, together with turkey coronavirus and pheasant coronavirus form group 3 gamma within the genus Coronavirus (Cavanagh et al., 2005). IBV virus contains three major structural proteins one of which

*Corresponding author. E-mail: r.toroghi@rvsri.ac.ir; phone: +98915-503-8022.

Abbreviations: $\mathrm{EID}_{50}=$ median embryo infectious dose, $\mathrm{HVR}=$ hypervarible region; IB = infectious bronchitis; IBV = infectious bronchitis virus; $\mathrm{LAV}=$ live attenuated vaccine; $\mathrm{pi}=$ post inoculation; $\mathrm{SPF}=$ specific pathogen free is spike (S) glycoprotein (Cavanagh, 2007; Jackwood et al., 2010). The spike protein is cleaved into two subunits, S1 (92K glycoprotein) and S2 (84K glycoprotein) (Casais et al., 2003; Jackwood et al., 2001). This protein is very variable commonly differing by $20 \%$ to $25 \%$ (Cavanagh, 2007; Liu et al., 2007). Infectious bronchitis (IB) is among the most common poultry diseases and it is difficult to control due to the presence of serotype and genotype variations as a result of differences in S1 gene of the virus which plays key role in virus-neutralizing antibodies, cell attachment and serotype specificity (Bijlenga et al., 2004; Huang and Wang, 2007). In Iran, IB is one of the most important respiratory diseases of broiler chicken flocks and its periodic disease outbreaks result in great financial losses to the poultry industry (Seyfi Abad Shapouri et al., 2004; Shirzad et al., 2012). Live attenuated vaccine (LAV) is the primary method of controlling IBV infection in commercial chickens (Ignjatovic and Sapats, 
2000; Ladman et al., 2006). To develop a LAV, IBV strains are passaged in specific pathogen free (SPF) embryonated chicken eggs to achieve a reduction in virulence for the respiratory and urinary tracts (Britton et al., 2012; Huang and Wang, 2007). It is demonstrated that most changes in the virus genome due to mutation and recombination during serial passaging in embryonated chicken eggs occur in the S1 gene. Some of these changes in S1 might be related to virus attenuation and host adaptation (Britton et al., 2012; Wang et al., 1995). LAV vaccines are generally applied for day-old chickens (Tarpey et al., 2006). Vaccines have generally been effective in controlling clinical signs associated with IBV infection, however, the emergence of new serotypes or variants may cause clinical disease in vaccinated flocks. New serotypes may arise either as a result of spontaneous mutation or recombination so that differences of 2 to $3 \%$ of S1 protein can change serotype (Bijlenga et al., 2004; Liu et al., 2007). It is known that modest cross protection occurs between different serotypes of IBV (Bijlenga et al., 2004; Jackwood et al., 2003). Therefore, it is not surprising that attempts to vaccinate commercial chickens with currently available vaccines might be unsuccessful when serotype of a vaccine is different from that of a dominant circulating serotype (Jackwood et al., 2003; Malo et al., 1998). That is what has happened in the recent emergence of 793/B serotype. This serotype caused severe problems in Massachusettsvaccinated chicken flocks in Europe and many other parts of the world (Malo et al., 1998). Massachusetts type vaccines are only partially effective against the serotype 793/B (Parsons et al., 1992). Therefore, it is advisable to develop a new LAV which can combat the dominant circulating serotype. It is the case in which a commercial 793/B serotype vaccine was developed by passaging the field virus in chicken embryonated eggs (Bijlenga et al., 2004). We demonstrated that 793/B serotype was the most dominant field circulating serotype in Iran in year 2008, while the Massachusetts type vaccine was the only vaccine used before 2006 (Shoushtari et al., 2008). Long-term circulation of the virulent virus and lack of a suitable vaccine caused great economic losses to Iranian poultry industry and lead to extensive 793/B commercial vaccine importation. At the moment, the Massachusetts vaccine along with 793/B vaccine is frequently used in our poultry industry. It was demonstrated that Iranian 793/B isolates had some critical amino acid changes compared to the foreign ones (Salarpour, et al., 2019). Due to these amino acid changes we expect that the vaccine developed by native isolates would be more efficacious. Hence, it is very valuable to develop a native LAV using our local 793/B isolates to enhance protection against the field virus. Currently, no data are available about the required passage levels for the development of a 793/B LAV. According to the studies concerning different IBV serotypes, including GA98 serotype, L1148 strain of QX-like IBV and a Taiwan group I strain of
IBV, attenuated viruses were achieved after 70, 80 and 74 passage levels, respectively (Geerligs et al., 2011; Huang and Wang, 2006). Geerligs et al. (2011) mentioned that passage levels around 80 might be adequate to attenuate many IBV serotypes. Therefore, in this study, we attempted to show that whether the virus passage levels around 80 in SPF chicken eggs could be considered as a threshold passage level for attenuation of the three Iranian isolates of 793/B serotype. Indeed, the serial passage of the viruses was more than the mentioned threshold, it was passage level 90. To show the degree of attenuation, we have also compared the passage levels 15 and 90 . Another part of this study was focused on identifying nucleotide and amino acid changes of $\mathrm{S} 1$ gene of the viruses during virus passaging. Despite several studies, mutations responsible for IBV attenuation have never been found (Britton et al., 2012; Wang et al., 1995). There are a few studies on genetic changes of S1 gene in 793/B serotype during virus passaging but except one nucleotide and amino acid change, other changes were not common between the studies. A change in amino acid 95 was identified by both Cavanagh and Callison in 739/B serotype after virus passaging (Callison et al., 2001; Cavanagh, 2005, 2007). In this paper, we have detected certain changes in S1 in our virus isolates after virus passage 90 .

\section{Materials and Methods}

Viruses. Three IB 793/B virus isolates (IR/773/2001, IR/794/2002 and IR/520/2002) were used for serial passaging. The isolates were isolated from broiler chicken flocks in our department previously (Momayez et al., 2002).

Virus passaging. Virus passages and titrations were performed on SPF chicken eggs (Venkateshwara Hatcheries, India). Serial passages were made up to passage 90 by inoculation of 10-day-old embryonated SPF chicken eggs via chorioallantoic sac route, each with a $0.2 \mathrm{ml}$ of the virus. All of dead embryos within the first $24 \mathrm{~h}$ of incubation were discarded. The chorioallantoic fluid was harvested from the surviving eggs two days post inoculation (pi), pooled and then dispensed in small aliquots. The portions were partly used for subsequent passages and the rest stored at $-70^{\circ} \mathrm{C}$ for further use. For subsequent passages the viruses were diluted 1:10 in phosphatebuffered saline. Stock viruses belonging to passagaes 15 and 90 were made. Accession numbers of viruses were as follows: MH252985 (IR/773/2001), MH252986 (773 passage 15), MH252987 (773 passage 90), MH236618 (IR/794/2002), MH252983 (794 passage 15), MH252984 (794 passage 90), MH252990 (IR/520/2002). Viruses of passage levels 15 and 90 were tested for bacteria, fungi, mycoplasma, and viruses including hemagglutinating viruses, Reovirus and chicken anemia virus using different methods like isolation, PCR and RT-PCR.

$R T$-PCR. To verify the IB isolates, viral RNA was extracted every 10 passages using the High pure viral nucleic acid kit (Roche, Ger- 
many), according to the manufacturer's instructions. Viral RNA was used in a reverse transcriptase-PCR (RT-PCR) using SuperScript ${ }^{\oplus}$ II Reverse transcriptase kit )Invitrogen, USA) in which the RNA was transcribed into cDNA under the conditions of $60 \mathrm{~min}$ at $42^{\circ} \mathrm{C}$ followed by $15 \mathrm{~min}$ at $70^{\circ} \mathrm{C}$. PCR was performed using Expand high fidelity PCR system enzyme (Roche) in reaction containing $10 \mathrm{x}$ buffer for Taq and Tgo enzymes containing $15 \mathrm{mM} \mathrm{MgCl}_{2}, 15 \mathrm{pM}$ forward and reverse primer, $20 \mu \mathrm{M}$ dNTPs, $3 \mu \mathrm{l}$ of cDNA, $2.6 \mathrm{U}$ of Taq and Tgo enzymes, nuclease free distilled water filled up to $50 \mu \mathrm{l}$ of final volume; with following conditions: initial denaturation step for $3 \mathrm{~min}$ at $94^{\circ} \mathrm{C}, 35$ cycles of $1 \mathrm{~min}$ at $94^{\circ} \mathrm{C}, 1.5 \mathrm{~min}$ at $45^{\circ} \mathrm{C}$ followed by the step of $3 \mathrm{~min}$ at $72^{\circ} \mathrm{C}$ and the final extension of $10 \mathrm{~min}$ at $72^{\circ} \mathrm{C}$. The universal published primer sequences used were 5'-TGAAACTGAACAAAAGAC-3' (Cal2) as forward primer and 5'-CATAACTAACATAAGGGCAA-3' (Cal1) as reverse primer (Callison et al., 2001). The nested PCR was performed using 5'-AGTAGTTTTGTGTATAAACCA-3' forward and 5'-ACATTAG TAATAAAACCTTG-3' as reverse primers (Adzhar et al., 1997).

Virus titration. Virus titration was done for passage levels 15 and 90 of each virus. Each dilution of the viruses was inoculated into three embryonated SPF chicken eggs. After 6 days of incubation, the embryos were examined for the presence of specific lesions including dwarfing, curling and stunting. Dead embryos and live embryos showing specific lesions were considered positive for IBV. The titre, expressed as the median embryo infectious dose $\left(E_{50}\right)$ per millilitre, was calculated according to the method of Spearmann-Karber (Finney, 1964).

Experimental design. Hundred days old SPF chicks were randomly divided into six groups, including 773(P15), 773(P90), 794(P15), 794(P90), positive control (793/B commercial vaccine) and negative control (uninfected). The degree of virulence of the viruses at two passage levels, P15 and P90, was determined by inoculating of the viruses into chicks at one day of age via occulonasal route at titres described in Table 1 . In virus titration, titres of isolate 520 at passage levels 15 and 90 were $10^{2.2}$ and $10^{2.9} \mathrm{EID}_{50} / \mathrm{ml}$, respectively. Since the increase in titer of this isolate at passage level 90 was not adequate enough to use as a vaccine seed, it was omitted from our study. The inoculated titer of passage level 15 of virus isolates 773 and 794 were adjusted to $10^{3}$ and $10^{2} \mathrm{EID}_{50} / \mathrm{bird}$, respectively; these titers were different from those adjusted for pas-

Table 1. Inoculated virus titers

\begin{tabular}{ccl}
\hline No. of birds & EID $_{\mathbf{5 0}}$ /bird & Virus \\
\hline 20 & $10^{3}$ & 773 passage $15(773 \mathrm{P} 15)$ \\
20 & $10^{3.6}$ & 773 passage $90(773 \mathrm{P} 90)$ \\
20 & $10^{2}$ & 794 passage $15(794 \mathrm{P} 15)$ \\
20 & $10^{3.6}$ & 794 passage $90(794 \mathrm{P} 90)$ \\
11 & $10^{3.6}$ & 793/B commercial vaccine \\
8 & - & negative control group \\
\hline
\end{tabular}

Inoculated $\mathrm{EID}_{50} /$ bird for each group was selected based on the calculated $\mathrm{EID}_{50} / \mathrm{ml}$ in virus titration test. Number of birds in each group is shown. sage 90 and vaccine control group. This was because the volume of inoculum could not exceed $250 \mu$ l. In passage level 90, the titer of 773(P90) and 794(P90) were adjusted according to 793/B commercial vaccine titer $\left(10^{3.6} \mathrm{EID}_{50} / \mathrm{bird}\right)$ to rationally compare our viruses at passage level 90 with the vaccine virus.

Pathogenicity test for respiratory tract and kidney infection in chickens. Pathogenicity testing was conducted according to section 113.327, d, 2, of title 9 of the code of federal regulations (Service, 1999). Pathogenicity test was performed to assess the degree of virulence of the viruses at passage 15 and 90 and included an assessment of cilliary activity of trachea (ciliostasis test), gross pathological examination of kidney and histopathological study of trachea and kidney samples. To do so, in each group, a quarter of birds in turn were humanly sacrificed at 5, 7 and 10 days pi and the remaining at 21 days pi. Samples of the trachea (lower part) and kidneys were collected and preserved in $10 \%$ formalin for further histopathological examination. Furthermore, sera were collected to perform ELISA (enzyme-linked immunosorbent assay).

Ciliostasis test. For ciliostasis test, the tracheas were collected aseptically, and placed in physiological saline buffer to remove mucin from the tracheal epithelial surface. From each trachea, 10 transverse rings of $0.6 \mathrm{~mm}$ were made. The ciliary activity of 3,4 and 3 sections of the upper, middle and lower parts of the trachea, respectively were determined by light microscopy at a magnification of 400x (Inverted microscope, Nikon, Japan). A score of 0 was given if the cilia in the whole tracheal section showed complete movement; score of 1 if the cilia of $67 \%$ to $100 \%$ of tracheal section showed movement; score of 2 if the cilia of $33 \%$ to $67 \%$ of the section showed movement; score of 3 if the cilia of less than $33 \%$ but not $0 \%$ of the section showed movement and a score of 4 if the cilia in the whole tracheal section showed no movement at all. For each trachea, the average ciliostasis score was calculated. The gross pathological examination was also performed on kidneys of each chicken used for the ciliostasis test.

Histopathology. Trachea and kidney samples in formalin were processed, embedded in paraffin, sectioned, and stained with haematoxylin and eosin. Trachea samples were observed for epithelial deciliation, epithelial hyperplasia and lymphoid infiltration. Kidney samples were studied for epithelial degeneration, ducto-tubular dilation, heterophil infiltration, lymphoid infiltration, epithelial regeneration, epithelial hyperplasia and lymphoid nodules. The severity index for each lesion was: - (negative) for no change, + (score 1 ) for mild change, ++ (score 2 ) for moderate change and +++ (score 3 ) for severe changes (Chen et al., 1996). The least significant difference of the means were statistically calculated by the Student's $t$-test using SAS software (SAS Institute, INC., USA).

Infectious bronchitis virus antibody test (Potency test). ELISA was performed with the sera collected at $5,7,10$, and 21 days pi using Infectious bronchitis virus antibody test kit (IDEXX, USA) to analyze the intensity and duration of antibody response in chickens. According to manufacturer's instruction, antibody titers higher than 396 were considered positive. Coefficient of variance percentage $(\% \mathrm{CV})$ was calculated for antibody titers in each group. 
Table 2. Ciliostasis test scores

\begin{tabular}{|c|c|c|c|c|c|c|c|c|c|}
\hline \multirow[b]{3}{*}{$\begin{array}{l}\text { Passage level } \\
\text { inoculated }\end{array}$} & \multicolumn{9}{|c|}{ Ciliostasis score } \\
\hline & \multicolumn{2}{|c|}{ Day 5 pi } & \multicolumn{2}{|c|}{ Day 7 pi } & \multicolumn{2}{|c|}{ Day 10 pi } & \multicolumn{2}{|c|}{ Day 21 pi } & \multirow[b]{2}{*}{ Total mean } \\
\hline & Individual & Mean & Individual & Mean & Individual & Mean & Individual & Mean & \\
\hline $773(\mathrm{P} 15)$ & $40,40,40,40,40$ & 40 & $40,40,40,40$ & 40 & $39,38,40,40$ & 39.25 & $29,28,27,27$ & 27.75 & 36.75 \\
\hline 773(P90) & $36,38,37,37,39$ & 37.4 & $38,37,39,39,39$ & 38.4 & $36,30,26,28$ & 30 & $12,6,9,4$ & 7.75 & 28.38 \\
\hline 794(P15) & $40,39,40,40,40$ & 39.8 & $40,40,40,40,37$ & 39.4 & $40,40,31,40$ & 37.75 & $28,27,24,27$ & 26.5 & 35.86 \\
\hline 794(P90) & $35,34,40,35,37$ & 36.2 & $40,35,40,11,40$ & 33.2 & $37,25,19,33,38$ & 30.4 & $8,8,0,6,0$ & 4.4 & 26.05 \\
\hline Positive control & $3,2,1$ & 2 & $0,2,1$ & 1 & $2,0,2$ & 1.3 & 0,2 & 1 & 1.32 \\
\hline Negative control & 0,0 & 0 & 0,0 & 0 & 0,0 & 0 & 0,0 & 0 & 0 \\
\hline
\end{tabular}

Results of ciliostasis tests on test groups, including those inoculated with 773(P15), 773(P90), 794(P15), 794(P90), 793/B commercial vaccine and a noninfected control group. In groups 773(P15), 773(P90) and 794(P15); 3, 2 and 2 birds died at 4 days pi, respectively.

Amplification of the S1 gene and sequencing. Isolate IR/520/2002 was discarded from study. RT-PCR was done as previously described. The resulting cDNA was amplified by PCR. PCR (as previously described) was performed to amplify the whole $S 1$ gene of the passage levels 15 and 90 viruses of isolates IR/773/2001 and IR/794/2002. PCR products with the approximate size of 1720 bp were purified using High pure PCR products purification kit (Roche), according to the manufacturer's instructions. Fifty $\mu \mathrm{l}$ of purified PCR products along with $20 \mu \mathrm{l}$ of each primer Call and Cal2 (Callison et al., 2001), SX1 and SX2 (Adzhar et al., 1997) and their complementary primers were sent to Macrogen Company (South Korea) for sequencing.

Sequence analysis. Attained sequences using mentioned primers were assembled by CLC main workbench version 5.5 and final S1 sequences were defined. Sequences of wild viruses and their passages 15 and 90 of isolates IR/773/2001 and IR/794/2002 were aligned and also translated to protein sequences using CLC main workbench version 5.5 and finally nucleotide and amino acid changes during virus passaging were analyzed.

\section{Results}

\section{RT-PCR detection test}

To confirm the presence of the IBV, we have provided RTPCR. All passages which were tested for detection of the IB viruses were positive in the RT-PCR, and a clear visible PCR product was observed in the agarose gel with the expected size (464 bp) (data not shown). In contamination testing, all samples were negative for extraneous organisms.

\section{Passaging and titration of the viruses}

Serial passaging of three isolates were made until passage level 90 with the perspective to attenuate our viruses. Virus titration was done on passage levels 15 and 90 of each virus isolate. The titer of each virus calculated based on $\mathrm{EID}_{50} / \mathrm{ml}$ were as follows: $10^{3.6}$ for $773(\mathrm{P} 15), 10^{5.2}$ for $773(\mathrm{P} 90), 10^{2.6}$ for $794(\mathrm{P} 15), 10^{4.6}$ for $794(\mathrm{P} 90), 10^{2.2}$ for $520(\mathrm{P} 15)$ and $10^{2.9}$ for 520 (P90).

\section{Pathogenicity test for respiratory tract and kidneys}

Clinical signs and gross lesions were studied to observe the changes in of pathogenicity of IBV in different passages. Coughing was the main clinical sign observed in birds during the infection. In groups inoculated with 773(P15), 773(P90) and 794(P15); 3, 2 and 2 birds died at 4 days pi, respectively. Gross pathological signs in dead birds included thick mucus in the trachea, pale and swollen kidneys with severe urate deposition. On gross examination of kidney of other birds, some cases had pale and swollen kidneys with mild urate deposition in the urethra (execpt 2 control groups). There was a decrease in the severity of the lesions in the kidneys of birds inoculated with the viruses of passage level 90 compared to viruses of passage 15 . But there was no difference in the severity of gross lesions between chicken groups of 773(P15) with 794(P15) and 773(P90) with 794(P90).

The maximum ciliostasis score was 40 , observed in 773(P15) at 5 and 7 days pi. Passage levels 90 in both isolates had lower ciliostasis scores compared to passage level 15 and a sharp decline in scores was observed at 21 days pi. In group 794(P90), there were great differences among individual values, including one chick at 7 days pi with score 11 and two chicks at 10 days pi with score 25 and 19 , which had much lower ciliostasis scores compared to other members of their group. Overall, ciliostasis scores decreased from day 5 to day 21 pi as expected. No ciliostasis was observed in the negative control group and a very mild ciliostasis observed in the positive control group. Results of assessment of ciliary activity and ciliostasis scores are shown in Table 2.

Microscopic lesions in trachea included epithelial deciliation, epithelial hyperplasia and lymphoid infiltration. The 
Table 3. Average severity score of histopathological lesions of trachea in each test group

\begin{tabular}{|c|c|c|c|c|c|c|c|}
\hline & Days post & 773(P15) & 773(P90) & 794(P15) & 794(P90) & $\begin{array}{c}\text { Positive } \\
\text { control }\end{array}$ & $\begin{array}{c}\text { Negative } \\
\text { control }\end{array}$ \\
\hline & & \multicolumn{6}{|c|}{ Mean } \\
\hline \multirow{4}{*}{ Epithelial deciliation } & 5 & 3 & 3 & 3 & 3 & 0 & 0 \\
\hline & 7 & 3 & 3 & 3 & 2.6 & 0.66 & 0 \\
\hline & 10 & 3 & 2.75 & 3 & 2.6 & 0 & 0 \\
\hline & 21 & 1.75 & 0.75 & 1.75 & 0.6 & 0.5 & 0 \\
\hline \multirow{4}{*}{ Epithelial hyperplasia } & 5 & 1 & 1 & 1 & 1 & 0.33 & 0 \\
\hline & 7 & 2 & 1.8 & 1.8 & 2 & 0.66 & 0 \\
\hline & 10 & 3 & 2.75 & 2.75 & 2.8 & 0.33 & 0 \\
\hline & 21 & 3 & 3 & 3 & 3 & 1 & 0 \\
\hline \multirow{4}{*}{ Lymphoid infiltration } & 5 & 0.4 & 0.4 & 0.6 & 0.4 & 0.66 & 0 \\
\hline & 7 & 0 & 0.6 & 0.4 & 0.6 & 0 & 0 \\
\hline & 10 & 1 & 0.25 & 0.5 & 0.4 & 0 & 0 \\
\hline & 21 & 0 & 0 & 0 & 0 & 0 & 0 \\
\hline
\end{tabular}

highest score for each lesion was 3. Score 3 of epithelial deciliation was found at 5 days pi in both passages of our two isolates. Passage level 15 of both isolates showed score 3 until day 10 pi and score 1.75 at day 21 pi. In groups 773(P90) and 794(P90) epithelial deciliation score decreased to scores 0.75 and 0.6 , respectively, at 21 days pi. Average score of epithelial deciliation in the positive control group was zero at 5 and 10 days pi and below score 1 at 7 and 21 days pi. Epithelial hyperplasia increased from score 1.8 to 3 for two passages of two isolates from day 5 to day $21 \mathrm{pi}$. The positive control group showed very mild epithelial hyperplasia, which reached score 1 at 21 days pi. In 773(P15) and 773(P90) groups, lymphoid infiltration was seen in $35.3 \%$ and $33.3 \%$ of birds, respectively, with the score of 1 while other birds in these groups were negative. In birds inoculated with 794(P15) and 794(P90), score 1 for lymphoid infiltration was found in $16 \%$ and $27.7 \%$ of birds, respectively, and score 2 in $11 \%$ and $5.5 \%$ of birds, respectively. In the positive control group, lymphoid infiltration was only found at 5 days pi. Negative control chickens were normal in all cases. Average of severity index for tracheal lesions for each test group are shown in Table 3. The differences of epithelial deciliation at days 5, 7 and 10 compared to day 21 pi were statistically significant in both passages of two isolates $(P \leq 0.05)$. In groups 773 (P90), 794(P15) and 794(P90) differences of epithelial hyperplasia at day 5 compared to day 7 pi were statistically significant. Also, the difference of epithelial hyperplasia was significant at days 7 and 10 pi between each group, 773(P15), 773(P90), 794(P15), 794(P90) compared with vaccine. Tracheal lesions are shown in Fig. 1. Microscopic tracheal lesions in dead chicks were hyperemia, blooding, severe deciliation and very mild epithelial hyperplasia.

Microscopic lesions in kidney included epithelial degeneration, ducto-tubular dilation, heterophil infiltration, lymphoid infiltration, epithelial regeneration, epithelial hyperplasia and lymphoid nodules. Except lymphoid nodule, all other lesions had average scores below score 1 with some exceptions, including score 1 for lymphoid infiltration in group 794(P90) at 10 days pi and the positive control group at 5 days pi, and score 1.8 for epithelial hyperplasia in group 794(P15) at 5 days pi. Lymphoid nodule was the most common microscopic finding in the kidney. It was observed as follows: $88 \%$ of birds in 773 (P15), 83\% in 773(P90), 89\% in 794(P15), 90\% in 794(P90) and 36\% in the positive control group. The highest score for this lesion was score 1.25. Scores above 2 were not seen in any of the kidney lesions. Epithelial hyperplasia was the only lesion which showed statistically significant difference between some groups $(P \leq 0.05)$. Those gropus included: 794(P15) and 794(P90) at day 5 pi, 794(P90) at days 5 and 7 pi; and 794(P15) and 773(P15) at day 5 pi. The negative controls were normal. Average of severity index for kidney lesions for each test group are shown in Table 4 and kidney lesions are shown in Fig. 2. Microscopic lesions
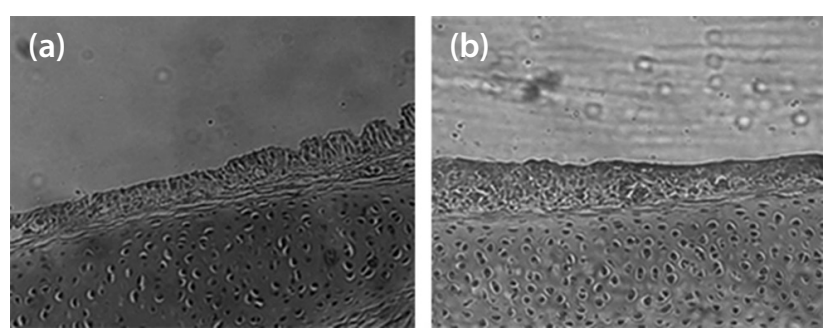

Fig. 1

Histopathology of trachea

(a) Histopathology of trachea. Epithelial deciliation and mild (score 1) epithelial hyperplasia. (b) Epithelial deciliation and severe (score 3) epithelial hyperplasia. 
Table 4. Average severity score of histopathological lesions of kidney in each test group

\begin{tabular}{|c|c|c|c|c|c|c|c|}
\hline & Days post & $773(\mathrm{P} 15)$ & 773(P90) & 794(P15) & 794(P90) & $\begin{array}{c}\text { Positive } \\
\text { control }\end{array}$ & $\begin{array}{c}\text { Negative } \\
\text { control }\end{array}$ \\
\hline & & \multicolumn{6}{|c|}{ Mean } \\
\hline \multirow{4}{*}{ Epithelial degeneration } & 5 & 0 & 0.8 & 0 & 0.2 & 0 & 0 \\
\hline & 7 & 0 & 0.2 & 0.2 & 0.2 & 0 & 0 \\
\hline & 10 & 0 & 0 & 0 & 0.2 & 0 & 0 \\
\hline & 21 & 0 & 0 & 0 & 0 & 0 & 0 \\
\hline \multirow{4}{*}{ Ducto-tubular dilation } & 5 & 0 & 0 & 0.4 & 0.2 & 0 & 0 \\
\hline & 7 & 0.25 & 0.2 & 0.2 & 0.4 & 0 & 0 \\
\hline & 10 & 0 & 0 & 0.5 & 0.2 & 0 & 0 \\
\hline & 21 & 0.25 & 0 & 0 & 0 & 0 & 0 \\
\hline \multirow{4}{*}{ Heterophil infiltration } & 5 & 0 & 0 & 0.2 & 0.8 & 0 & 0 \\
\hline & 7 & 0 & 0 & 0 & 0 & 0.33 & 0 \\
\hline & 10 & 0 & 0 & 0.25 & 0.2 & 0 & 0 \\
\hline & 21 & 0 & 0 & 0 & 0 & 0 & 0 \\
\hline \multirow{4}{*}{ Lymphoid infiltration } & 5 & 0.8 & 0.2 & 0.4 & 0.4 & 1 & 0 \\
\hline & 7 & 0.75 & 0 & 0.6 & 0.4 & 0.66 & 0 \\
\hline & 10 & 0.5 & 0.75 & 0.25 & 1 & 0.66 & 0 \\
\hline & 21 & 0.25 & 0 & 0 & 0.2 & 0.5 & 0 \\
\hline \multirow{4}{*}{ Epithelial regeneration } & 5 & 0 & 0 & 0 & 0 & 0 & 0 \\
\hline & 7 & 0 & 0 & 0 & 0 & 0 & 0 \\
\hline & 10 & 0 & 0 & 0.25 & 0 & 0 & 0 \\
\hline & 21 & 0.25 & 0 & 0.25 & 0 & 0 & 0 \\
\hline \multirow{4}{*}{ Epithelial hyperplasia } & 5 & 0 & 0.2 & 1.8 & 0 & 0.66 & 0 \\
\hline & 7 & 0 & 0.4 & 0.4 & 0.8 & 0 & 0 \\
\hline & 10 & 0 & 0 & 0.5 & 0.2 & 0.33 & 0 \\
\hline & 21 & 0 & 0 & 0.75 & 0.6 & 0 & 0 \\
\hline \multirow{4}{*}{ Lymphoid nodules } & 5 & 0.6 & 0.6 & 1 & 0.8 & 0 & 0 \\
\hline & 7 & 1.25 & 0.8 & 1 & 1 & 0 & 0 \\
\hline & 10 & 1 & 1 & 1 & 1.2 & 0.66 & 0 \\
\hline & 21 & 1 & 1 & 1 & 0.8 & 1 & 0 \\
\hline
\end{tabular}

in the kidneys of dead chicks included severe hyperemia, blooding, tubulitis and nephritis.

\section{Infectious bronchitis virus antibody test}

ELISA was performed to analyze the intensity and duration of antibody response in chickens. Based on ELISA results, inoculated groups showed a negative antibody response at days 5 and 7 pi except group 773(P90) which was also negative at even 10 days pi. In other inoculated groups, some samples were positive at 10 days pi. At 21 days pi, all inoculated chicks were positive. All of the sera in the negative control group were negative. Average antibody titers in all inoculated chicks (except group 794 (P90)) increased from day 10 to 21 pi and they were as follows: 1375 to 5590 in 773(P15), 347 to 978 in 773(P90), 1113 to 1642 in 794(P15), 1199 to 1120 in 794(P90) and 440 to 2335
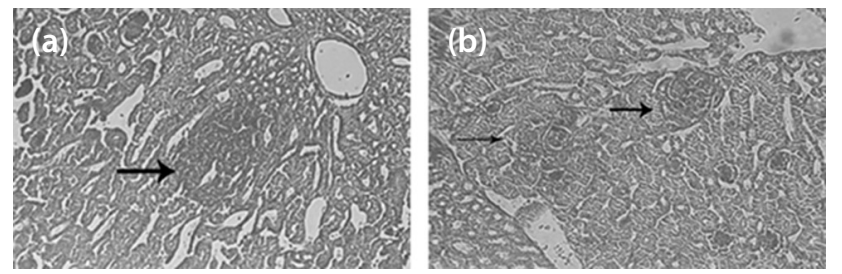

Fig. 2

Histopathology of kidney

(a) Focal lymphoid nodule. (b) Multifocal lymphoid nodule.

in the positive control group. In most cases \%CV showed variable responses except 773 (P90) at days 10 and 21 pi with $\% \mathrm{CV}$ of $11 \%$ and $5 \%$, respectively, which had more uniform titers than other groups. Results from days 10 and 21 pi are shown in Table 5. 
Table 5. Antibody titres at days 10 and 21 pi based on ELISA

\begin{tabular}{|c|c|c|c|c|c|c|}
\hline \multirow{3}{*}{$\begin{array}{l}\text { Passage level } \\
\text { inoculated }\end{array}$} & \multicolumn{6}{|c|}{ Antibody titer } \\
\hline & \multicolumn{3}{|c|}{ Day $10 \mathrm{pi}$} & \multicolumn{3}{|c|}{ Day 21 pi } \\
\hline & Individual & Mean & $\% \mathrm{CV}$ & Individual & Mean & $\% \mathrm{CV}$ \\
\hline $773(\mathrm{P} 15)$ & $1939,377,1833,1353$ & 1375 & 52 & $4664,6606,6655,4435$ & 5590 & 21 \\
\hline 773(P90) & $377,298,338,377$ & 347 & 11 & $918,945,1032,1081$ & 978 & 5 \\
\hline $794(\mathrm{P} 15)$ & $326,1968,221,1939$ & 1113 & 87 & $1339,1368,1894,1968$ & 1642 & 20 \\
\hline 794(P90) & $594,2945,377,111,1968$ & 1199 & 100 & $720,1300,876,691,2015$ & 1120 & 50 \\
\hline Positive control & $260,326,734$ & 440 & 60 & 4046,621 & 2335 & 100 \\
\hline
\end{tabular}

Titers above 396 were considered positive according to manufacturer's instruction.

\section{Nucleotide sequence analysis}

S1 gene sequences of 773, 773(P15), 773(P90), 794, 794(P15) and 794(P90) were aligned and compared to find point mutations during virus passaging. The seqeunces of passage 15 and wild viruses were identical and no point mutation was found. All point mutations were seen at passage 90. Nucleotide positions of point mutations at passage 90 were as follows: $68,112,113,259,277,1314$ and 1424 in 773(P90); and 63, 70, 71, 110, 129, 152, 160, 163, 171, 220, 226, 259, 388, 419, 424, 478, 490, 492, 511, 675, 857, 873,
893, 911, 915, 966, 981, 1023, 1145, 1178, 1179, 1290, 1314, 1476 and 1561 in 79(P90). Nucleotide difference between passage 15 and passage 90 was $0.43 \%$ and $2.16 \%$ in 773 (P90) and 794(P90) respectively.

\section{Amino acid sequence analysis}

At the next step all of the nucleotide sequences were translated to amino acid sequences to find amino acid substitutions. Amino acid sequence alignment is shown in Fig. 3. Based on amino acid sequence alignment some point

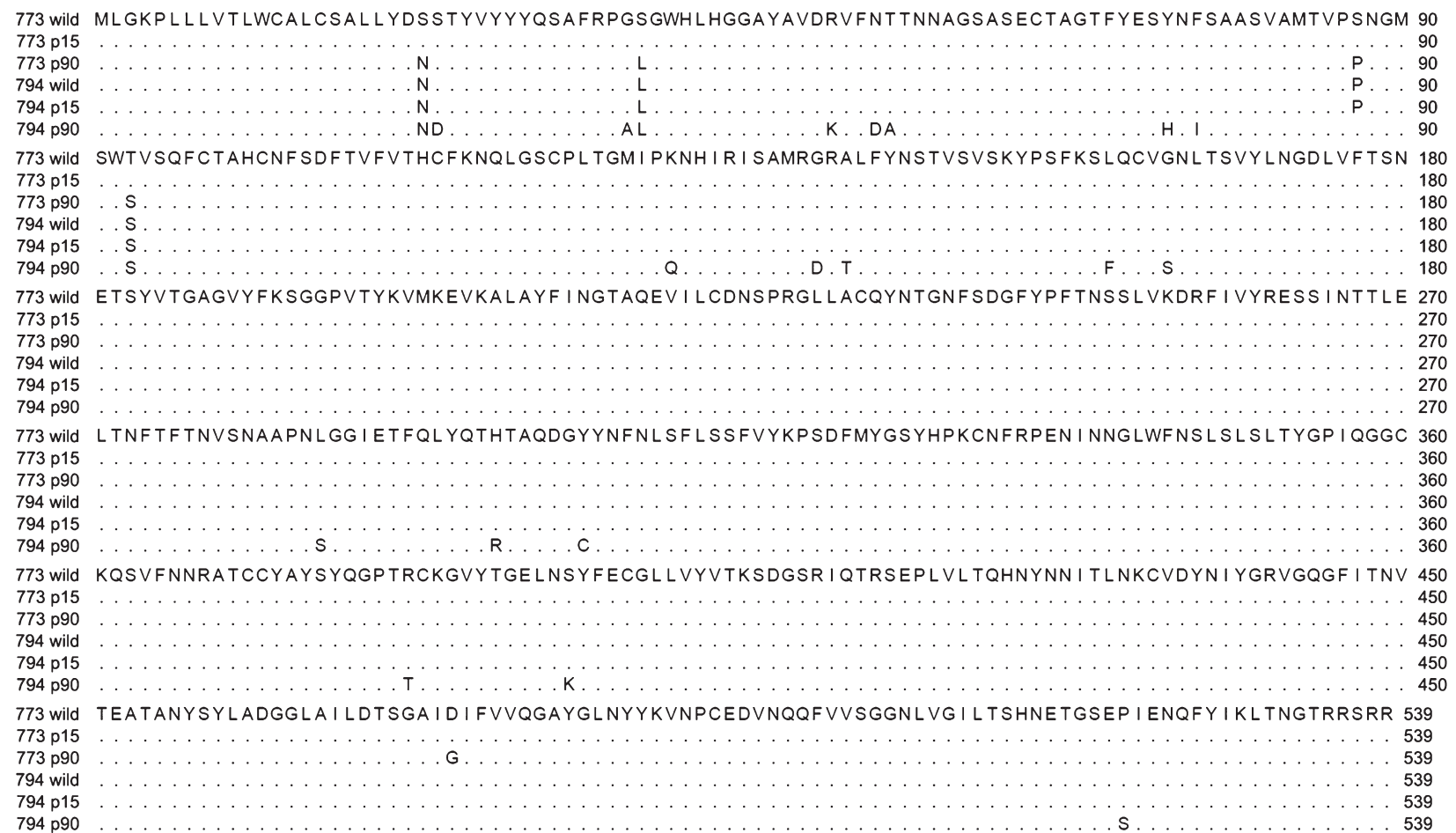

Fig. 3

Alignment of deduced S1 amino aicd sequences of 773 and 794 isolates

Alignment was done to compare pasages 15 and 90 of isolates 773 and 794 (773 p15; 773 p90; 794 p15; 794 p90) with the wild type viruses (773 wild; 794 wild). 
Table 6. Amino acid changes in isolates 773 and 794 during virus passaging

\begin{tabular}{|c|c|c|c|c|c|c|c|}
\hline 773(P90) & 773(P15) & 773 wild type & aa position & 773(P90) & 773(P15) & 773 wild type & Amino acid position \\
\hline S & $\mathrm{T}$ & $\mathrm{T}$ & 93 & $\mathrm{~N}$ & $S$ & S & 23 \\
\hline \multirow[t]{2}{*}{ G } & $\mathrm{D}$ & $\mathrm{D}$ & 475 & $\mathrm{~L}$ & S & S & 38 \\
\hline & & & & $\mathrm{P}$ & S & S & 87 \\
\hline 794(P90) & 794(P15) & 794 wild type & aa position & 794(P90) & 794(P15) & 794 wild type & Amino acid position \\
\hline $\mathrm{T}$ & A & A & 142 & $\mathrm{D}$ & $S$ & $S$ & 24 \\
\hline $\mathrm{F}$ & $\mathrm{L}$ & $\mathrm{L}$ & 160 & A & G & G & 37 \\
\hline S & G & G & 164 & $\mathrm{~K}$ & $\mathrm{R}$ & $\mathrm{R}$ & 51 \\
\hline S & $\mathrm{L}$ & $\mathrm{L}$ & 286 & $\mathrm{D}$ & $\mathrm{N}$ & $\mathrm{N}$ & 54 \\
\hline $\mathrm{R}$ & $\mathrm{H}$ & $\mathrm{H}$ & 298 & A & $\mathrm{T}$ & $\mathrm{T}$ & 55 \\
\hline $\mathrm{C}$ & $\mathrm{Y}$ & $\mathrm{Y}$ & 304 & $\mathrm{H}$ & $\mathrm{Y}$ & $\mathrm{Y}$ & 74 \\
\hline $\mathrm{T}$ & $\mathrm{R}$ & $\mathrm{R}$ & 382 & I & $\mathrm{F}$ & $\mathrm{F}$ & 76 \\
\hline $\mathrm{K}$ & S & S & 393 & S & $\mathrm{P}$ & $\mathrm{P}$ & 87 \\
\hline \multirow[t]{2}{*}{ S } & $\mathrm{P}$ & $\mathrm{P}$ & 521 & $\mathrm{Q}$ & $\mathrm{K}$ & $\mathrm{K}$ & 130 \\
\hline & & & & $\mathrm{D}$ & G & $\mathrm{G}$ & 140 \\
\hline
\end{tabular}

mutations were silent (synonymous substitution). Amino acid differences were seen at positions 23, 38, 87, 93 and 475 in 773(P90); and positions $24,37,51,54,55,74,76,87$, $130,140,142,160,164,286,298,304,382,393$ and 521 in 794(P90). Amino acid variation between passage 15 and passage 90 was $0.93 \%$ and $3.53 \%$ in isolates 773 and 794 , respectively. List of amino acid differences in each isolate are listed in Table 6.

\section{Discussion}

Control of infectious bronchitis is partially achieved by using live attenuated vaccines (Cavanagh, 2005). Due to the large number of antigenic types of the virus with no or weak cross-protection, the choice of vaccine serotype should be carefully considered. When current vaccines cannot produce enough protection against a dominant circulating IBV serotype, it is advisable to use other suitable commercial vaccines or otherwise, develop a live attenuated vaccine with a dominant circulating IBV serotype in the region (Cavanagh, 2003; Cook et al., 1999). Knowing that 793/B serotype is one of the most dominant circulating serotypes in Iran, we focused on attenuation of its isolates (IR/773/2001 and IR/794/2002) by serial passaging in SPF chicken eggs. We demonstrated that Iranian 793/B isolates had some amino acid changes compared to the foreign one (Salarpour et al., 2019). Those changes, considered as amino acid markers for Iranian 793/B isolates, were located at variable regions of the S1 protein containing epitopes which play role in inducing neutralizing antibodies. Due to the critical location of those amino acid differences, we expect better efficacy of vaccines developed from Iranian isolates compared to imported ones. Attenuation of GA98 virus, L1148 strain of QX-like IBV and A Taiwan group I IBV showed that the viruses were adequately attenuated at passage levels 70, 80 and 74, respectively (Geerligs et al., 2011; Huang and Wang, 2006). Geerligs et al. (2011) declared that passage level 80 would be enough to suitably attenuate many IBV serotypes. Therefore, we considered passage level 80 as a threshold and continued passages to level 90 to make sure that our viruses become sufficiently attenuated. The attenuation degree of our viruses was assessed using potency and pathogenicity tests, including ciliostasis test and histopathology for two passage levels 15 and 90 .

Although three local IB isolates were selected for serial passages, isolate IR/520/2002 had a low increase in titer at passage $90\left(10^{2.9} \mathrm{EID}_{50} / \mathrm{ml}\right)$ and was removed from the study. Titers of isolates 773 and 794 at passages level 15 and 90 increased during serial passaging $\left(10^{3.6}\right.$ to $10^{5.2} \mathrm{EID}_{50} / \mathrm{ml}$ for 773 isolate and $10^{2.6}$ to $10^{4.6} \mathrm{EID}_{50} / \mathrm{ml}$ for 794 isolate). This was an indication of virus adaptation to its new host. In the study by Jackwood et al. (2003) on GA98 serotype, titer of the virus was $7.3 \times 10^{7} \mathrm{EID}_{50} / \mathrm{ml}$ for passage level 7 . This is much more than the amount that we found in this study in passage 90 . This primary evidence showed that our viruses might not be adequately adapted and attenuated. To do

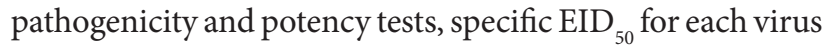
was inoculated to each bird. The titer of passage 90 of the two isolates was adjusted similarly to $793 / \mathrm{B}$ commercial vaccine $\left(10^{3.6} \mathrm{EID}_{50} / \mathrm{bird}\right)$. Regarding to the limitation of inoculation volume $(250 \mu \mathrm{l})$, the highest possible titers to inoculate to birds for passage 15 of 773 and 794 isolates were $10^{3}$ and $10^{2}$ $\mathrm{EID}_{50} /$ bird, respectively.

Ciliostasis test was performed following virus inoculation of chicks at one day of age. The results demonstrated a mild decrease in virulence of isolates 773 and 794 at passage level 90 compared to passage level 15. Moreover, based on cili- 
ostasis scores, isolate 773 showed more virulence at both passages compared to isolate 794 . As expected, ciliostasis scores decreased from day 5 to day 21 pi and the decrease was more significant at 21 days pi at passage level 90 . The total mean of ciliostasis scores of passages 15 and 90 of virus isolate 773 ( 36.75 and 28.38, respectively) were a little bit higher than those in isolate 794 (35.86 and 26.05, respectively). Higher ciliostasis score of isolate 773 at passage level 15 (36.75) compared to that of isolate 794 (35.86) may be related to the higher inoculated titer of this virus $\left(10^{3} \mathrm{EID}_{50} /\right.$ bird for 773 against $10^{2} \mathrm{EID}_{50}$ /bird for 794). Since ciliostasis score of passage 90 of isolate 773 (28.38) was higher than passage 90 of isolate 794 (26.05), higher ciliostasis scores of isolate 773 at both passage levels might be the result of more virulent nature of this isolate compared to isolate 794. An average ciliostasis score of 20 has been indicated as threshold for virus attenuation (Geerligs et al., 2011; O.I.E. 2004). Despite the decrease of ciliostasis score during serial passaging, this score was still higher than the threshold at passage 90 for both isolates. In the study by Geerligs et al. (2011) on L1148 strain of QX-like IBV, ciliostasis score was below the threshold at passage level 80, which was a sign of adequate attenuation of the virus. However, the high ciliostasis score of passage 90 in our study may indicate the need for more passages.

In histopathology, severity of deciliation of tracheal epithelia mildly decreased from passage level 15 to passage 90. Epithelial deciliation and epithelial hyperplasia were the two most common histopathological findings in the trachea. After virus inoculation and over time, epithelial deciliation decreased, while epithelial hyperplasia increased. The epithelial deciliation severity index was the same for the two groups of 773(P15) and 794(P15). In these two groups, the highest score was at days 5, 7 and 10 pi (score 3 ) and it decreased to 1.75 at 21 day pi. In groups 773(P90) and 794(P90), epithelial deciliation score began to decrease sooner and at day $21 \mathrm{pi}$ they had score of 0.75 and 0.6 , respectively. It demonstrates that regeneration of the tracheal epithelium had been more rapid in chicks inoculated with passage 90 compared to passage 15 , which is a sign of lower virulence for chicks. Epithelial deciliation score in group 794(P90) decreased at day 7 pi while for group 773(P90), it decreased at day 10 pi. In this case, it could be a sign of higher virulence of 773(P90). Histopathology findings in the trachea were in line with ciliostasis test results.

During the pathogenicity test, in groups inoculated with 773(P15), 773(P90) and 794(P15); 3, 2 and 2 birds died during 4 days pi, respectively. Low severity of kidney lesions at passage 90 of both isolates would be a sign of low pathogenicity and more relative attenuation of the viruses. Although, some chicks died in the 773(P90) group, there were no losses in the 794(P90) group. This may be related to the higher virulence of isolate 773. In the gross pathology of surviving birds, we found no lesions.
In histopathology of kidney, lymphoid infiltration and lymphoid nodules were the most common findings compared to other lesions in the kidney. Lymphoid nodules were observed in focal or multifocal forms. In some birds, other lesions including epithelial degeneration, ducto-tubular dilation, heterophil infiltration, epithelial regeneration and epithelial hyperplasia were seen and their score was below the score 1 (mild). We found no clear correlation between severity of kidney lesions and the virulence of the virus. The same histopathology findings in the kidney were also observed in birds infected with nephropathogenic IBV strain but with more severity in the study by Chen et al. (1996). They have found all mentioned kidney lesions in birds infected with nephropathogenic IBV strain MA-87 (Chen et al., 1996).

Results of ELISA test demonstrated a decrease in virulence and immunogenicity of both isolates from passage 15 to pasaage 90. Positive antibody titres in sera appeared at day $10 \mathrm{pi}$ and all samples were positive at day $21 \mathrm{pi}$. Due to higher virulence and therefore higher immunogenicity of passage 15 , antibody titers were higher in chicks inoculated with passage 15 of our viruses. Antibody titer in group 773(P15) was higher than that in 794(P15). It could be because of higher virulence of the former isolate at passage 15 or higher titer of inoculated virus $\left(10^{3} \mathrm{EID}_{50} /\right.$ bird for 773 and $10^{2} \mathrm{EID}_{50} / \mathrm{bird}$ for 794). In contrast to the results of ciliostasis and histopathology tests, 773(P90) showed lower average antibody titer (978) compared to the titer of 794(P90) (1120). However, in the $773(90)$ group, \%CV was lower $11 \%$ and $5 \%$ at 10 and 21 days pi, respectively, than that in the 794(90) group with $100 \%$ and $50 \%$ at 10 and 21 days pi, respectively. In study on GA98 serotype vaccine by Jackwood et al. (2003) antibody titers were extremely low at 5 and 10 days after inoculation of virus passage 80 and were not statistically different from the negative controls. We also found negative titers until day 10 pi in some birds. Since research studies and field findings demonstrate that we are not able to track antibody titers until day $10 \mathrm{pi}$, therefore, ELISA test before day $10 \mathrm{pi}$ is not recommended. Although we have not studied the cell mediated immunity (CMI), there is possibility to find variation in inducing CMI among IBV serotypes.

Based on our results, it seems that 773(P90) and 794(P90) have not been attenuated adequately to use as an vaccine seed. However, it was shown that virulance of the viruses has reduced during serial passages. Unlike previous studies which showed attenuation of many IBV serotypes occurred around passage 80 , we found that our isolates, even up to the passage 90 , retained some degree of virulance. A study by Chen et al. on nephropathogenic IBV strain MA-87 showed that the virus became attenuated at passage level 110 (Chen et al., 1996). Also Lee et al. (2010) obtained attenuated virus of a nephropathogenic strain called K2/01 after 170 passages (Lee et al., 2010). It is clear that nephropathogenic IBV strains may need more passage levels to be attenuated compared to 
IBV strains which mainly attack the respiratory tract. So it is inferred that our viruses require more than 100 passages to be sufficiently attenuated. Due to the gradual decrease in the virulance of our viruses (773 and 794) from the passage 15 to 90 , it seems that a sufficiently attenuated virus will be obtained in higher passage levels. Indeed, virus passages should continue to obtain an optimum balance between the adequate virulence and the capacity to induce protective immunity along with high virus titer (Britton et al., 2012). Overall, it can be concluded that there is no threshold passage level for IBV attenuation not only among the different serotypes, but also among different strain of a specific IBV serotype. Because of this variation, it is advisable to select and use several field isolates for vaccine seed development simultaneously and suitable passage level for attenuation should be determined empirically.

Also in current study nucleotide and amino acid changes in $\mathrm{S} 1$ of passaged viruses were compared. This protein is very variable and plays key role in virus-neutralizing antibodies, cell attachment and serotype specificity (Cavanagh, 2007; Gelb et al., 2005; Huang and Wang, 2007; Liu et al., 2007). S1 protein has three hypervarible regions (HVR) with their location in European strains as follows: HVR1 (aa 56-69), HVR2 (aa 117-131) and HVR3 (aa 274-387) (Montassier, 2010). On the other hand, Cavanagh et al. located HVR1 and HVR2 at amino acid positions 38-51 and 99-115 respectively by studying seven Massachusetts strains (Cavanagh et al., 1988).

Each of our isolates underwent different nucleotide changes at passage 90 . There was no difference between wild viruses and their passage 15 and all changes were seen from passage 15 to passage 90 . In comparison to wild viruses, 773(P90) and 794(P90) showed 5 and 19 amino acid changes which calculates to $99.07 \%$ and $96.47 \%$ identities, respectively. Some mutations changed the amino acid type. These included polar serine to hydrophobic leucine and proline, and charged aspartic acid to hydrophobic glycine at residues 38, 87 and 475 respectively in isolate 773 , and, polar threonine to hydrophobic alanine, hydrophobic proline to polar serine, hydrophobic glycine to charged aspartic acid, hydrophobic alanine to polar threonine, hydrophobic glycine to polar serine, hydrophobic leucine to polar serine and hydrophobic proline to polar serine at residues 55,87 , $140,142,164,286,521$ respectively in isolate 794 .

Some of the changes included substitution of polar and charged amino acids with hydrophilic residues or vice versa. Therefore, some of these alterations could remarkably change the properties and structure of the S1 protein. Hydrophilic residues have tendency to be located nearer to the exterior surfaces of a protein and thereby are more accessible and can react with other molecules, while hydrophobic residues locate in the interior. Charged amino acids are involved in electrostatic and polar amino acids in hydrogen bonds. Both electrostatic and hydrogen bonds play role in antigen and antibody reactions. Therefore, changing amino acids from polar or charged to nonpolar has potential to cover some parts of epitopes. Substitution of hydrophilic amino acids with polar or charged one can disclose some part of protein that might be an epitope. Moreover, changing amino acids can change binding properties of protein to its receptor or virus adaptation to a specific host (Cavanagh et al., 1988).

Glycine and proline are unique amino acids. Although glycine is a hydrophobe amino acid but its side chain is so tiny that it has no role in hydrophobic reaction therefore it is seen on the surface of proteins and loop regions. It makes proteins flexible. Proline mostly locates in interior of protein and contributes to protein rigidity. Thanks to the properties mentioned before, these two proteins are conserved (Cavanagh et al., 1988). Substitution of amino acids with glycine can be remarkable because glycine resides in loop regions and also gives flexibility to the region and has both properties for epitope including accessibility and flexibility. In isolate 773, amino acid position 475 has changed from aspartic acid to glycine. And, vice versa in isolate 794 amino acid positions 37, 140 and 164 have changed from glycine to alanine, aspartic acid and serine, respectively.

In both isolates, amino acid position 87 has changed but in isolate 773 from serine to proline and in 794 from proline to serine. Since serine is polar and proline is nonpolar this region attains potential to locate in the interior part of the protein in isolate 773 and in the exterior part in isolate 794 at passage 90 .

In the current study most of the amino acid changes located at positions 23-55, 74-93 and 130-164. Among them region $23-55$ has some overlap with HVR1 position introduced by Cavanagh. Some of the changes observed in our study located at HVR regions introduced by Montassier and Cavanagh including HVR1 residues 38 in isolate 773 and 51 in isolate 794 for HVR1 and 131 in isolate 794 for HVR2 and 286, 298, 304 and 382 in isolate 794 for HVR3. Considering many studies done on different serotypes of IBV, it is demonstrated that most of the amino acid changes reside within 300 amino acids from the beginning of the protein (Ammayappan et al., 2009; Cavanagh et al., 1992; Kant et al., 1992). In our study, we found similar results, most of the changes located at residues 23-298 except residues 475 in isolate 773 and 304, 382, 393 and 521 in isolate 794. Wang et al. (1995) found an antigenic determinant that was highly conserved and located at amino acid position 250-255 (Wang et al., 1995). We also found region with no amino acid change.

Cavanagh et al. (2005) found a change in amino acid 95 after 10 passages in 793/B serotype. In another study, Callison et al. (2001) found that three nucleotide changes were in attenuated 793/B virus including nucleotides 283, 1522 and 1589. Change in amino acid 95 (nucleotide 283) was common in the studies done by Cavanagh and Callison. This 
amino acid is serine in field isolates of 793/B serotype and alanine in attenuated viruses. It was suggested that the change in nucleotide 283 is related to the replication of the virus in the host (chicken or chicken embryo) and not related to the pathogenicity of the virus (Cavanagh, 2005, 2007). Comparing with previous studies on nucleotide changes of serotype 793/B during virus passaging, none of the changes reported were seen in the current study. It is not clear that why this amino acid did not change in our viruses after 90 passages. It raised a question whether this amino acid substitution is really related to replication of the virus in each host. It is probable to see change in amino acid 95 in our viruses at higher passage levels.

Based on our results and some other studies, we found that each researcher identified different mutations in the S1 gene. Our two isolates showed different mutations and different virulence compared to each other. Based on our findings we could not find relationship between amino acid changes and the pathogenicity of our viruses. We assume that attenuation might not be directly related to mutations in S1 gene during virus passaging; or there are some other mutations in other genes responsible for attenuation. Also, special attention should be payed to natural selection of a specific virus subpopoulation during serial passaging of IBVs instead of detecting the point mutations. In contrast to many IBV serotypes, our viruses were not adequately attenuated at passage 90 . All these findings clearly demonstrate that IBV serotypes and even isolates of the same serotype differ in mutations, virulence, antigenicity and replication.

Acknowledgments. This work was fully supported by Ministry of Agriculture - Jahad, Razi Vaccine and Serum Research Institute.

\section{References}

Adzhar A, Gough RE, Haydon D, Shaw K, Britton P, Cavanagh D (1997): Molecular analysis of the 793/B serotype of infectious bronchitis virus in Great Britain. Avian Pathol. 26, 625-640. https://doi.org/10.1080/03079459708419239

Ammayappan A, Upadhyay C, Gelb JR, Vakharia VN (2009): Identification of sequence changes responsible for the attenuation of avian infectious bronchitis virus strain Arkansas DPI. Arch Virol. 154, 495-499. https://doi. org/10.1007/s00705-009-0325-9

Bijlenga G, Cook JK, Gelb JR, de Wit JJ (2004): Development and use of the $\mathrm{H}$ strain of avian infectious bronchitis virus from the Netherlands as a vaccine: a review. Avian Pathol. 33, 550-557. https://doi.org/10.1080/03079450400013154

Britton P, Armesto M, Cavanagh D, Keep S (2012): Modification of the avian coronavirus infectious bronchitis virus for vaccine development. Bioeng Bugs. 3, 114-119. https:// doi.org/10.4161/bbug. 18983

Callison SA, Jackwood MW, Hilt DA (2001): Molecular characterization of infectious bronchitis virus isolates foreign to the
United States and comparison with United States isolates. Avian Dis. 45, 492-499. https://doi.org/10.2307/1592994 Casais R, Dove B, Cavanagh D, Britton P (2003): Recombinant avian infectious bronchitis virus expressing a heterologous spike gene demonstrates that the spike protein is a determinant of cell tropism. J. Virol. 77, 9084-9089. https://doi. org/10.1128/JVI.77.16.9084-9089.2003

Cavanagh D (2003): Severe acute respiratory syndrome vaccine development: experiences of vaccination against avian infectious bronchitis coronavirus. Avian Pathol. 32, 567-582. https://doi.org/10.1080/03079450310001621198

Cavanagh D (2005): Coronaviruses in poultry and other birds. Avian Pathol. 34, 439-448. https://doi.org/10.1080/0307 9450500367682

Cavanagh D (2007): Coronavirus avian infectious bronchitis virus. Vet Res. 38, 281-297. https://doi.org/10.1051/ vetres:2006055

Cavanagh D, Davis PJ, Cook JK, Li D, Kant A, Koch G (1992): Location of the amino acid differences in the S1 spike glycoprotein subunit of closely related serotypes of infectious bronchitis virus. Avian Pathol. 21, 33-43. https:// doi.org/10.1080/03079459208418816

Cavanagh D, Davis PJ, Mockett AP (1988): Amino acids within hypervariable region 1 of avian coronavirus IBV (Massachusetts serotype) spike glycoprotein are associated with neutralization epitopes. Virus Res. 11, 141-150. https:// doi.org/10.1016/0168-1702(88)90039-1

Cavanagh D, Picault JP, Gough R, Hess M, Mawditt K, Britton P (2005): Variation in the spike protein of the 793/B type of infectious bronchitis virus, in the field and during alternate passage in chickens and embryonated eggs. Avian Pathol. 34, 20-25. https://doi.org/10.1080/03079450400025414

Chen BY, Hosi S, Nunoya T, Itakura C (1996): Histopathology and immunohistochemistry of renal lesions due to infectious bronchitis virus in chicks. Avian Pathol. 25, 269-283. https://doi.org/10.1080/03079459608419141

Cook JK, Orbell SJ, Woods MA, Huggins MB (1999): Breadth of protection of the respiratory tract provided by different live-attenuated infectious bronchitis vaccines against challenge with infectious bronchitis viruses of heterologous serotypes. Avian Pathol. 28, 477-485. https://doi. org/10.1080/03079459994506

Finney DJ (1964): The spearman-Karber method. In Finney DJ (Ed.): Statistical Methods in Biological Assays. Charles Griffin, London, pp. 524-530.

Geerligs HJ, Boelm GJ, Meinders CA, Stuurman BG, Symons J, Tarres-Call J, Bru T, Vila R, Mombarg M, Karaca K, Wijmenga W, Kumar M (2011): Efficacy and safety of an attenuated live QX-like infectious bronchitis virus strain as a vaccine for chickens. Avian Pathol. 40, 93-102. https://doi.org/10.1080/03079457.2010.542742

Gelb JR, Weisman Y, Ladman BS, Meir R (2005): S1 gene characteristics and efficacy of vaccination against infectious bronchitis virus field isolates from the United States and Israel (1996 to 2000). Avian Pathol. 34, 194-203. https:// doi.org/10.1080/03079450500096539

Huang YP, Wang CH (2006): Development of attenuated vaccines from Taiwanese infectious bronchitis virus strains. 
Vaccine 24, 785-791. https://doi.org/10.1016/j.vaccine.2005.08.081

Huang YP, Wang CH (2007): Sequence changes of infectious bronchitis virus isolates in the $3^{\prime} 7.3 \mathrm{~kb}$ of the genome after attenuating passage in embryonated eggs. Avian Pathol. 36, 59-67. https://doi.org/10.1080/03079450601110015

Ignjatovic J, Sapats S (2000): Avian infectious bronchitis virus. Rev. Sci. Tech. 19, 493-508. https://doi.org/10.20506/ rst.19.2.1228

Jackwood MW, Hilt DA, Brown TP (2003): Attenuation, safety, and efficacy of an infectious bronchitis virus GA98 serotype vaccine. Avian Dis. 47, 627-632. https://doi. org/10.1637/6094

Jackwood MW, Hilt DA, Callison SA, Lee CW, Plaza H, Wade E (2001): Spike glycoprotein cleavage recognition site analysis of infectious bronchitis virus. Avian Dis. 45, 366-372. https://doi.org/10.2307/1592976

Jackwood MW, Hilt DA, Sellers HS, Williams SM, Lasher HN (2010): Rapid heat-treatment attenuation of infectious bronchitis virus. Avian Pathol. 39, 227-233. https://doi. org/10.1080/03079451003801516

Kant A, Koch G, van Roozelaar DJ, Kusters JG, Poelwijk FA, van der Zeijst BA (1992): Location of antigenic sites defined by neutralizing monoclonal antibodies on the $S 1$ avian infectious bronchitis virus glycopolypeptide. J. Gen. Virol. 73, 591-596. https://doi.org/10.1099/0022-1317-73-3-591

Ladman BS, Loupos AB, Gelb JR (2006): Infectious bronchitis virus $\mathrm{S} 1$ gene sequence comparison is a better predictor of challenge of immunity in chickens than serotyping by virus neutralization. Avian Pathol. 35, 127-133. https:// doi.org/10.1080/03079450600597865

Lee HJ, Youn HN, Kwon JS, Lee YJ, Kim JH, Lee JB, Park SY, Choi IS, Song CS (2010): Characterization of a novel live attenuated infectious bronchitis virus vaccine candidate derived from a Korean nephropathogenic strain. Vaccine 28, 2887-2894. https://doi.org/10.1016/j.vaccine.2010.01.062

Liu S, Han Z, Chen J, Liu X, Shao Y, Kong X, Tong G, Rong J (2007): $S 1$ gene sequence heterogeneity of a pathogenic infectious bronchitis virus strain and its embryo-passaged, attenuated derivatives. Avian Pathol. 36, 231-234. https://doi. org $/ 10.1080 / 03079450701338730$

Malo A, Orbell S, Huggins M, Woods M, Cook J (1998): Cross protection studies after the use of live attenuated IBV vaccines Nobilis ${ }^{\oplus}$ IB 4-91 and Nobilis ${ }^{\oplus}$ IB Ma5 (Massachusetts type). Poultry $17,1-4$.
Momayez R, Pourbakhsh SA, Khodashenas M, Banani M (2002): Isolation and identification of infectious bronchitis virus from commercial chickens. Arch. Razi Ins. 53, 1-9.

Montassier HJ (2010): Molecular epidemiology and evolution of avian infectious bronchitis virus. Rev. Bras. Cienc. Avic. 12, 87-95. https://doi.org/10.1590/S1516$\underline{635 X 2010000200003}$

O.I.E. (2004): Manual of Standards of Diagnostic Tests and Vaccines, pp. 561-69.

Parsons D, Ellis MM, Cavanagh D, Cook JK (1992): Characterisation of an infectious bronchitis virus isolated from vaccinated broiler breeder flocks. Vet. Rec. 131, 408-411. https://doi.org/10.1136/vr.131.18.408

Salarpour A, Toroghi R, Momayez R (2019): Nucleotide sequence analysis of $\mathrm{S} 1$ gene among iranian avian infectious bronchitis viruses isolated during 2001 - 2002. Arch. Razi. Ins. 74, 21-31.

Service AapHI: (1999). Title 9, Code of federal regulations, standard requirements for IBV vaccines. Animal and Plant Health Inspection Service. Washington, D.C., The United States.

Seyfi Abad Shapouri MR, Mayahi M, Assasi K, Charkhkar S (2004): A survey of the prevalence of infectious bronchitis virus type 4/91 in Iran. Acta Vet. Hung. 52, 163-166. https:// doi.org/10.1556/AVet.52.2004.2.4

Shirzad MR, Asasi K, Mohammadi A (2012): Efficacy of vaccination programmes using two commercial live infectious bronchitis vaccines against a field IRFIB 32 strain. Bulg. J. Vet. Med. 15, 260-272.

Shoushtari AH, Toroghi R, Momayez R, Pourbakhsh SA (2008): 793/B type, the predominant circulating type of avian infectious bronchitis viruses 1999-2004 in Iran: A retrospective study Arch. Razi. Ins. 63, 1-5.

Tarpey I, Orbell SJ, Britton P, Casais R, Hodgson T, Lin F, Hogan E, Cavanagh D (2006): Safety and efficacy of an infectious bronchitis virus used for chicken embryo vaccination. Vaccine 24, 6830-6838. https://doi.org/10.1016/j.vaccine.2006.06.040

Wang L, Parr RL, King DJ, Collisson EW (1995): A highly conserved epitope on the spike protein of infectious bronchitis virus. Arch. Virol. 140, 2201-2213. https://doi.org/10.1007/ $\underline{\text { BF01323240 }}$ 